\title{
Comunicación

\section{Libros para los wixáritari. Una historia de integración, exclusión y emancipación}

Books for wixáritari. A history of integration, exclusion and emancipation

La autora analiza la especificidad de los textos dirigidos a indígenas wixáritari a partir de un censo de libros construido para este fin. Las editoriales, fechas, tirajes, género y lengua de los libros, en un contexto histórico de políticas editoriales para indígenas en México, le permiten concluir sobre la meta integradora del wixárika al Estado nacional a la vez que se le excluye de la creación editorial dirigida a ellos mismos. Se comentan algunos libros dialógicos que pueden cumplir con un propósito emancipatorio.

PALABRAS CLAVE: Libros en lenguas indígenas, historia de libros wixáritari, escritura y políticas públicas.
The author of this article discusses the books published for Wixaritari. She analyzes the specificity of these texts from a survey of books built for this purpose. In a historical context of editorial policy for indigenous people in Mexico, the publishers, dates, gender and language of the books, show the books' objective to integrate the indigenous people to the national state whilethey are excluded from the editorial decisions. Some books that meet dialogic and emancipatory purposes are mentioned.

KEY WORDS: Books in indigenous languages, history of Wixaritari books, writing and public policy.

1 Universidad de Guadalajara, México.

Correo electrónico: corona.berkin@gmail.com

Parres Arias 150, Piso 1, Col. Belenes, C.P. 45100; Zapopan, Jalisco, México. 
En el presente artículo me intereso por las políticas editoriales dirigidas a los wixáritari, donde solo hablaré de los libros dirigidos $a$ lectores wixáritari y no de los libros sobre los wixáritari, cuyos lectores en primer lugar son los no wixáritari. En este censo de libros mi corpus suma 55 libros publicados de 1906 a la fecha, en su propia lengua o de forma bilingüe. Además de los casos en los que se explicita que el libro es para lectores wixáritari, considero los libros escritos en su lengua, ya que muestran una intención de dirigirse a lectores competentes de la lengua. Al presentar el panorama editorial de libros para los wixáritari, a partir de las editoriales que han publicado para ellos, los tirajes, las lenguas en la que están escritos, las fechas y los géneros abordados, es posible aproximarse a responder la pregunta que guía esta investigación: ¿los libros editados para los wixáritari son estrategias para facilitar la integración nacional, son formas de excluirlos de la comunicación escrita o son vías de emancipación?

En el primer apartado resumo una discusión en torno a las funciones sociales del libro y la escritura, y una reflexión con relación a su adopción en América Latina; enseguida presento una síntesis de las políticas editoriales educativas oficiales dirigidas a indígenas en México. En el caso de los indígenas wixáritari -dada la ausencia de una historia formal de la aparición de la escritura en su lengua- expongo algunos hechos relevantes en torno a la creación de su escritura y su estandarización. Esta investigación se encuentra basada en el análisis del corpus de libros desde las siguientes categorías: editoriales, fechas, tirajes, género y lengua.

\section{¿EL LIBRO A PRUEBA DE LAS CULTURAS?}

Desde la perspectiva educativa se contempla el proceso de lectoescritura como una vía de acceso a la cultura. La promoción de la lectura, el aprendizaje del descifrado y sus estrategias para desarrollar una escritura correcta, los planteamientos gramaticales o literarios, son posturas que apuestan al aprendizaje de habilidades técnicas para el desarrollo intelectual.

Pero en una posición claramente política se considera que aprender a leer y escribir no es solo repetir signos; alfabetizar es aprender herra- 
mientas que faciliten el pensamiento y la acción críticos. De esta manera se propone enseñar la escritura a partir de la historia, necesidades propias y la situación social.

Desde una perspectiva crítica de la escritura, Claude Lévi-Strauss (1992) describe su función como potenciadora del poder. Para el autor la escritura no se reduce a una técnica informativa y menos a una herramienta para el desarrollo de la humanidad; considera que los grandes saltos cualitativos de la existencia humana se deben a la observación, experimentación y transmisión de los hallazgos y no a su registro escrito. Si bien acepta que la escritura amplifica la aptitud de los hombres para preservar los conocimientos, no ve que esta memoria artificial haya desarrollado la conciencia del hombre para aprender del pasado ni para organizar el presente y el futuro de las culturas occidentales.

Lo que sí acompaña a la escritura es la urbanización y con ella la división clara entre los que saben escribir y los que no saben. La primera Constitución del Estado de Jalisco, publicada en 1824, legislaba el vínculo entre la escritura y la ciudadanía: "El ejercicio de los derechos de ciudadanía se suspenden ... Por no saber leer y escribir; pero esta disposición no tendrá efecto hasta después del año de 1840". Si bien es claro que en esa fecha no se contemplaba a los indígenas en la definición de ciudadanos, nos interesa la escritura por su facultad para participar en los asuntos políticos. Las características de la escritura primero y de la imprenta después, marcan la conformación de una sociedad letrada, jerarquizada, supeditada a las exigencias de unas reglas gramaticales correctas.

Ángel Rama (1984) nos propone una historia de la función de la escritura en los países latinoamericanos a partir de la Conquista. La Corona se preocupó por mantener un grupo social alfabetizado que tuviera a su cargo prolongar el orden de la monarquía en América. Estos, al servicio del Rey, controlarían la jerarquía y el orden del universo a partir de los signos de la nueva cultura. Así, los letrados -nuevos criollos americanos- se convirtieron en poseedores de "un alto ministerio que los equiparaba a una clase sacerdotal" (p. 23), a cargo de dos quehaceres: la enorme tarea administrativa colonial y la exigencia de evangelización de los indígenas; ambas tareas reclamaban "sacerdotes" alfabetizados. 
Los criollos se encargaron de este poder que servía al Rey y a la vez los volvía dueños del lenguaje simbólico de las nuevas tierras, ya que desde la letra escrita no solo se dominó en la burocracia legal sino también el mundo cultural: se diferenciaron las celebraciones cultas de las populares, ${ }^{2}$ se modelaron la ciudades con sus calles, nombres y números, se definieron los sistemas educativos, y los de ocio y entretenimiento.

Atrincherados en el centro de la Colonia, se agruparon, institucionalizaron y conformaron un grupo urbano burocrático que controlaría desde la capital el resto de los territorios. La ciudad triunfó así sobre el campo y el centro sobre las regiones, de forma que perdura hasta la fecha: la escritura articula su relación con el poder, lo sustenta, justifica y construye una distancia entre los alfabetizados y los de la cultura oral, los que dictan las políticas desde el centro y los que las acatan desde lo local.

El libro, producto de la cultura occidental, ¿puede cumplir con otros objetivos que no sean los que responden a la lógica disciplinante de la que nacieron?, ¿qué podemos comprender a partir de los libros que son explícitamente editados para los wixáritari?

\section{LOS WIXÁRITARI CON RELACIÓN AL LIBRO}

Y A LA ESCRITURA

La educación indígena en el Occidente del país y la enseñanza del castellano es tan antigua como la Colonia (Castañeda, 1984). Sin embargo, hablar de la escritura y el libro dirigido a los wixáritari se remonta en nuestro corpus a 1906, fecha del primer libro encontrado en esta lengua: un catecismo, el cual puede ser considerado tardío en un sentido, pero novedoso en otro. El primer catecismo en lengua náhuatl data de 1528 , por lo que el catecismo para wixáritari se puede considerar de reciente aparición. Por otro lado es el primer intento de escribir y publicar un texto en la lengua wixárika.

2 Véase Jesús Martín-Barbero (1987) con referencia al teatro culto basado en la escritura a diferencia del melodrama que en su origen encuentra la prohibición de la palabra en las representaciones populares y por lo tanto la necesidad de un exceso de gestualidad. 
Por otro lado, en México, la Secretaría de Educación Pública (SEP) tiene una presencia decisiva con relación a la edición de libros para indígenas. En nuestro corpus, 322 de los 55 títulos que existen para wixáritari son ediciones o coediciones con la SEP. El proyecto nacional adquiere ciertas características propias según el momento histórico; sin embargo, se puede observar una constante: el interés durante los más de 90 años que lleva de operar la SEP, de castellanizar y alfabetizar a la población indígena. Florescano (1999) señala: "Para construir a la nueva nación se unifica la lengua en primer lugar y en seguida el sistema educativo" (p. 278). Los libros de educación del Estado mexicano han sido el vehículo para construir el ideal de un mexicano mestizo y alfabetizado, donde los indígenas son el mayor obstáculo para un país con vocación a la modernidad. 4

En la discusión en torno a la mejor manera de educar a los indígenas -cuestión ampliamente debatida en la SEP desde su fundación- José Vasconcelos ${ }^{5}$ tuvo una posición muy definida. En cuanto a la creación de escuelas especiales para indígenas, manifestó:

... siempre he sido enemigo de esta medida porque fatalmente conduce al sistema llamado "de la reservación", que divide la población en castas y colores de la piel, y nosotros deseamos educar al indio para asimilarlo totalmente a nuestra nacionalidad y no para hacerlo a un lado. En realidad creo que debe seguirse, para educar al indio, el método venerable de los grandes educadores españoles, que como Las Casas, Vasco de Quiroga y Motolinía, adaptaron al indio a la civilización europea, creando de esta manera nuevos países y nuevas razas, en lugar de borrar a los naturales o de reducirlos al aislamiento (Revista Educación,1923, p. 7).

3 El corpus consiste en los libros para wixáritari que la SEP ha publicado en 90 años y que se encuentran catalogados en Corona Berkin y De Santiago (2011); además se incluyen los títulos encontrados en los principales acervos especializados en libros en lenguas indígenas, así como en colecciones privadas.

4 La información que a continuación se ofrece sobre las publicaciones indígenas de la SEP se extrajo de Corona Berkin y De Santiago (2011) (Nota del editor: se puede consultar la reseña del libro en este número).

5 Primer ministro de la SEP e ideólogo de muchos de los programas educativos aún vigentes en México, quien fungió de 1921 a 1924. 
La enseñanza del idioma español se vio como la mejor estrategia para la unificación nacional, si bien no se rescató el interés de esos primeros misioneros por conocer los idiomas indígenas y menos aún atender a la preservación de sus culturas y sus diferencias.

En el periodo de 1924 a 1928, con Plutarco Elías Calles como presidente, continuó defendida la castellanización como vía única de educación para los indígenas, posición defendida por Moisés Sáenz y Rafael Ramírez. ${ }^{6}$ Se pensó que era difícil que prosperara la política educativa oficial de "enseñar a vivir" a los indígenas si antes no hablaban la lengua nacional. Por lo tanto en estos años no hubo libros en otras lenguas.

Sin embargo, Moisés Sáenz, el organizador de la escuela rural y propugnador de incorporar al indígena a la civilización, confesó más tarde en su vida, el fracaso de la experiencia que promovió. Tarde se dio cuenta que de forma unilateral y sin considerar la voz de los campesinos e indígenas, el proyecto educativo nacional no podría realizarse:

La vida cuaja en los moldes viejos. El débil reflejo de la escuela se pierde en la penumbra del subconsciente. Los maestros seguirán enseñando. Los gobiernos seguirán pagando escuelas. Esfuerzo y dinero se perderán como en un tonel sin fondo, a menos que haya un programa educativo más pleno y de mayor alcance y una filosofía social que obligue a la escuela a proyectarse definitivamente en la comunidad (Morales, 1986, p. 462).

\section{La educación socialista y el bilingüismo}

Durante el Maximato (1928-1934), años que se consideran continuidad de la presidencia de Calles, comenzaron a darse posiciones diferentes en lo concerniente a la educación de las comunidades indígenas. Por un lado Moisés Sáenz vio en la composición de la población mexicana,

6 Moisés Sáenz fue ministro de la SEP en la presidencia de Calles y subsecretario durante el Maximato. Rafael Ramírez fue el ideólogo de las escuelas rurales y proletarias en México; jefe de las Misiones Culturales de 1923 a 1924 y del Departamento de Escuelas Rurales en 1935. Ambos educadores fueron influenciados por el pensamiento positivista de Gabino Barreda y su proyecto de la educación científica como solución al retraso y la explotación del pueblo mexicano. 
con 14 millones de indios y mestizos y solo millón y medio de blancos, la persistencia de formas de vida primitiva en los indígenas que era necesario integrar a la civilización. Por otro lado, para el secretario Narciso Bassols7 la propuesta fue lograr una síntesis de las dos culturas:

Si hemos de triunfar, será porque logremos conservar la estructura espiritual de los indígenas, al mismo tiempo que los dotemos de los auxiliares insustituibles de la técnica científica ... Pero nos esforzamos por salvar del alma del indígena todas las virtudes en que, sin discusión sobrepasan a los tipos morales del capitalismo contemporáneo. Cuando se piensa en una cultura de síntesis como la que intentamos realizar, se obtiene una visión optimista del destino futuro de los indígenas, porque se contempla una organización social que conservará las valiosas fuerzas de disciplina, de cooperación, de armonía y laboriosidad que son características de las comunidades indígenas y que permitirán formar colectividades humanas mucho más robustas y valiosas que aquellas que han nacido de la lucha secular entre el egoísmo desenfrenado y las necesidades de unificación y organización social (citado en Labra, 1985, p. 48).

Durante el periodo de 1934-1940, con Lázaro Cárdenas en la presidencia de la República, se destacó la orientación educativa hacia las comunidades indígenas en la que ya se propugnó por el bilingüismo:

Los lingüistas del Instituto Lingüístico de Verano se unieron a profesionales de instituciones mexicanas para viajar entre grupos indios y llevar a cabo estudios de treinta idiomas vernáculos, formando un alfabeto basado en la fonética y la fonémica, elaborando gramáticas, diccionarios y listas de vocabulario, y aleccionando a los maestros en las técnicas de la educación bilingüe (Heath,1986, p. 171).

Pero pasó el tiempo antes de que estas acciones se manifestaran en publicaciones en idiomas vernáculos.

7 Narciso Bassols fue titular de la SEP de 1931 a 1934 y se caracterizó por las ideas en pro del socialismo y opuesto radicalmente a la participación del clero en la educación. 


\section{Civismo y amor patrio}

Con Manuel Ávila Camacho al frente del país entre 1940 y 1946, y con la entrada de México en la Segunda Guerra Mundial, se buscó reforzar el amor patrio. La formulación de una historia hecha por héroes y la propuesta de una nación homogénea excluía las múltiples culturas que habitaban el territorio.

De nuevo los pueblos indígenas no se reconocieron en las estrategias educativas y más bien, con el afán de unificación nacionalista se prepararon seis cartillas en lenguas indígenas. Estas consistían en lecciones de civismo nacional ilustradas con la flora y la fauna de cada localidad.

Torres Bodet ${ }^{8}$ (1946) se enfrentaba al problema de la integración indígena:

... menospreciar las particularidades de los núcleos indígenas equivaldría a condenarlos a una asimilación limitada, aleatoria, torpe e injusta. ¿Qué adhesión podría esperar el maestro de un niño yaqui, tarasco u otomí cuando -para radicar en él la mexicanidad que nos proponemos- se empeñara en ceñirle a las fronteras de un mundo abstracto, ajeno a sus inquietudes y a sus problemas, con citas de seres y de paisajes que nunca tuvo ocasión de ver? (p. 13).

Pero la propuesta novedosa no llegó más allá de utilizar los signos conocidos por los indígenas para imponer los significados de la mexicanidad y la unidad nacional.

\section{Desarrollo nacional}

El sexenio de Alemán (1946-1952) fue el de la escuela fundada en la idea de que el progreso explica el desarrollo de la cultura. La educación en general -y específicamente la tecnológica- era la vía hacia la producción económica; fueron los días de la escuela productiva, del aprender haciendo.

En cuanto a la educación indígena hay dos hechos de importancia administrativa. En 1947 desapareció el Departamento Autónomo

8 Torres Bodet, seguidor de la apuesta educativa de Vasconcelos, fungió como titular de la SEP de 1943 a 1946. 
de Asuntos Indígenas y lo remplazó como dependencia de la SEP la Dirección General de Asuntos Indígenas. Por lo menos en su nombre, cambiaba lo "autónomo" por lo "general", mostrando más claramente la realidad de esa oficina. Además, el 4 de diciembre de 1948 se creó el Instituto Nacional Indigenista (INI), con el que se coordinaría la SEP para asuntos relacionados con educación indígena. El año 1947 también fue el año en que se publicó la Primera Cartilla Huichol del Instituto Lingüístico de Verano (ILV), y la SEP el primer libro para wixáritari que se producía después de más de 30 años del catecismo de 1906.

Es momento en este lugar de hablar del ILV, ya que de 1947 y por 35 años, tuvo una exclusiva participación en la edición de libros para wixáritari. El ILV fue un organismo dedicado a la investigación de las lenguas indígenas con el objeto de traducir la Biblia. La filial mexicana del ILV fue fundada en México en 1936 por el ultraconservador estadounidense William Cameron Townsend (1896-1982); su contraparte mexicana, Moisés Sáenz, había estudiado en el Colegio Seminario Presbiteriano y admiraba a John Dewey. Ambos fueron apoyados por el gobierno de Lázaro Cárdenas.

Los lingüistas voluntarios eran capacitados durante los cursos de verano impartidos en varios lugares del mundo; de allí el nombre del Instituto. Los nuevos misioneros eran generalmente ingenuas parejas que estaban dispuestas, por muy poco dinero, a sacrificarse para ingresar a lugares geográficos inhóspitos y contactar a los pueblos indígenas más apartados. El ILV recibía apoyo de Nelson Rockefeller y la millonaria Standard Oil Company además de aportaciones filantrópicas y religiosas.

La relación entre el ILV y el gobierno mexicano era conveniente para este último, ya que se delegaban en el Instituto responsabilidades como la de alfabetizar, castellanizar y retirar del alcoholismo a los indígenas; además servían para pacificar a los indígenas e informar de sus actividades. Los misioneros lingüistas tenían pleno acceso dentro del territorio mexicano y disfrutaban de prestigio y reconocimiento. Entre otros, Joseph Grimes, uno de los miembros del ILV, elaboró los programas para el estudio de las lenguas indígenas en la Universidad Nacional Autónoma de México (UNAM) y Townsend recibió El Águila Azteca en 1978, la más alta condecoración que otorga el gobierno de México a los extranjeros. 
Hacia finales de los años 1970, el ILV recibió críticas nacionales e internacionales que señalaban cómo a través de Rockefeller se colonizaba económicamente a América Latina, mientras Townsend lo hacía desde la cultura y juntos colaboraban, por lo menos ideológicamente, con la política anticomunista estadounidense. Como se verá adelante, en 1979 el gobierno mexicano dio por terminada la relación con el ILV.

\section{Los libros de texto gratuitos}

De 1958 a 1964 hubo la voluntad política para alentar la educación al ofrecer a la infancia mexicana millones de libros de texto gratuitos para las escuelas primarias de todo el país. Dicha iniciativa es vigente hasta nuestros días.

Durante estos años el criterio dominante de la política nacional continuó con la "mestización" cultural pero se reconocieron oficialmente las contribuciones de las culturas indígenas, de forma que la SEP ratificó en 1963 la educación bilingüe:

... los maestros bilingües deberían introducir la lectura y la escritura en lenguas indígenas antes de alfabetizar en idioma nacional. Al año siguiente, esta Secretaría dio nueva vida al programa de alfabetización nacional que había sido suprimido durante la administración de Ruiz Cortines. ... la colaboración entre el personal del INI, la Secretaría de Educación Pública y, también, el Instituto Lingüístico de Verano, destacó mucho más que en los años anteriores. El Instituto Lingüístico de Verano preparaba cartillas y demás materiales de enseñanza y llevaba a cabo investigaciones lingüísticas entre las muchas lenguas que no habían sido analizadas y registradas alfabéticamente ... La Secretaría de Educación Pública administró las escuelas rurales y algunos programas indios especiales ... y llevó la responsabilidad principal en cuanto a la impresión de cartillas y materiales educativos empleados en las áreas indias (Heath, 1986, p. 226).

La participación del ILV en la zona wixárika en la década de 1960, consistió en publicar con la SEP tres cartillas más para los wixáritari; el ILV además publicó el Nuevo Testamento en wixárika. En 1969 la SEP determinó la desaparición de la Dirección General de Asuntos In- 
dígenas y la Dirección General de Internados de Primera Enseñanza y Educación Indígena, acentuando el fin del indigenismo en México.

\section{Los nuevos libros de texto gratuitos}

Con Luis Echeverría en el poder sobresalió la preparación de nuevos libros de texto para primaria y secundaria, pero para indígenas aparecieron solo cinco obras de reducidas dimensiones y propósitos muy diversos, producto de la colaboración entre la SEP y el ILV: textos bilingües para contar dinero o "leer la hora" del reloj.

La alfabetización de los indígenas no dejaba de interesar: "Conscientes de que no seremos una nación unificada mientras los mexicanos no hablemos el mismo idioma, en enero de 1974 se inició el programa nacional de castellanización" (Bravo,1976, p.120). Con base en métodos bilingües de contenido bicultural, se atendía a 300 mil niños de zonas indígenas de primero a sexto año. Con relación a los materiales para wixáritari, encontramos en toda esta década solo un título que de hecho es una reedición del ILV de una cartilla de 1965.

\section{Más libros para indigenas}

Con José López Portillo como presidente, en 1976, se estimó en 6 millones el número de adultos analfabetos; un millón de adultos indígenas que no hablaban español. El número absoluto de analfabetos había permanecido constante durante 50 años, por lo que se establecieron múltiples programas compensatorios, de los cuales se consideraron prioritarios: castellanizar y proporcionar la primaria bilingüe a la población indígena.

Este sexenio marcó el final de la colaboración con la SEP del ILV. Desde los estudios del Instituto de Investigaciones e Integración Social del estado de Oaxaca que demostraron las palpables deficiencias de las cartillas elaboradas por lingüistas del ILV, se habían acumulado críticas de diversos sectores al trabajo del Instituto. En 1979 el gobierno mexicano encontró que era más útil negarles las visas de estudio a los misioneros del ILV, que prolongar la relación. Por un lado, el Partido Revolucionario Institucional (PRI), a partir de sus redes de caciques y clientelismo asociado, había logrado penetrar en las áreas rurales y controlar los conflictos indígenas y el ILV no le era ya necesario; por otro 
lado, expulsarlos era un buen guiño con la izquierda mexicana. De esta manera el estudio de las lenguas indígenas pasó a manos de las instituciones públicas mexicanas y la SEP tomó en sus manos el tema de la educación indígena.

Así, vemos surgir una nueva política editorial para indígenas. Durante este sexenio apareció la serie Tradición Oral Indígena con seis libros bilingües de literatura náhuatl, wixárika y tzetzal: cuentos, canciones, leyendas, mitos y fiestas. El título en wixárika y español es de 1982 y es el primer libro después de las cartillas de alfabetización, el catecismo y el Nuevo Testamento. El libro es para niños y consiste en rescatar aspectos de la cultura oral wixárika.

Entre 1981 y 1983 se editó un libro de texto y un libro para el maestro; sin embargo, es importante señalar que estos libros no fueron utilizados por los maestros bilingües en la Sierra Huichola ya que la escritura aún no respondía a un sistema convencional que pudiera socializar sus contenidos.

\section{Modernización de la nación}

Al iniciar el sexenio 1988-1994, la preocupación del presidente Carlos Salinas de Gortari se enfocaba en que: “... menos del 3\% de la población escolar se orienta hacia carreras científicas. Esta tendencia, por sí sola, pone en riesgo la tarea de modernización nacional, así como el proyecto mismo del país en el siglo XXI." (Plan Nacional de Desarrollo,1989). Las prioridades fueron fortalecer el idioma nacional, las matemáticas y reformar la enseñanza de la historia por una historia que adecuara la identidad nacional a la globalización. En cuanto a los indígenas, no hay programa que proponga otra cosa que no sea traducir a lenguas indígenas los libros que provenían de comunidades rurales.

En su turno, el gobierno del presidente Ernesto Zedillo se vio obligado a definir sus prioridades. En el contexto de confrontación política y debilidad económica (devaluación de 3.30 a 6 pesos por dólar en febrero de 1995), puede verse la voluntad de negociación con los grupos indígenas en rebelión desde el 1 de enero de 1994, que llevó a la administración de Zedillo a firmar los llamados Acuerdos de San Andrés -si bien no los ratificaría después-, así como a trazar las líneas de su política social. 


\section{En palabras del Presidente:}

El aspecto más ambicioso de la política educativa en este sexenio reside en los llamados programas compensatorios. Estos programas hacen que para muchísimos niños y jóvenes, la educación efectivamente sea la diferencia entre una vida de marginación y pobreza, y una vida con oportunidades de mejoramiento (Zedillo, 1997).

En el debate sobre el rezago educativo en las zonas indígenas, lo que estaba en juego eran los alcances mismos de la aceptación de la diversidad. El mismo avance en la producción de textos escolares reflejaba que la política educativa para los indígenas mostró progresos en la comprensión de algunas de las necesidades de los diversos grupos étnicos. El panorama editorial para los wixáritari creció en número en esta década; además, en 1989 por vez primera se edita un libro monolingüe en wixárika distinto a una cartilla alfabetizadora o un libro religioso: la Antología Narrativa Huichola, de Paula Gómez y Xitákame Ramírez De la Cruz.

De 1990 a 1999 se publicaron 11 títulos nuevos realizados en coedición con editoriales como la Universidad de Guadalajara (UdeG); en 1991 se escribieron los libros para segundo y tercer grados de educación primaria en wixárika.

En su Memoria del Quehacer Educativo 1995-2000, Limón Rojas (2000) remarcó que la edición de libros de texto en lenguas indígenas fue constante:

Se aumentó la producción ..., ya que de 34 títulos existentes en 1994, logramos elaborar otros 153 en 55 variantes de 33 lenguas indígenas, de los que se modificaron 15 títulos, incorporando ejercicios que promuevan la participación del niño en los aspectos de indagación, creatividad y comunicación (p. 23).

Sin embargo, los libros supuestamente diseñados especialmente para cada grupo étnico, solo lo son por la lengua en que se editan, ya que tanto en los temas, la estructura y las ilustraciones todos los libros son homogéneos. 
En los cuadernos de trabajo de las instancias oficiales para la educación intercultural se pueden encontrar dibujos y fotografías. Ninguno de los dos tiene que ver ... con el grupo cultural al que van dirigidos, pues el criterio de selección es el tema, no el destinatario (Pérez-Daniel, 2010, p. 102).

En el año 2000, durante la debatida campaña electoral para la presidencia de la República, el tema de las deficiencias educativas fue central; sin embargo, ningún partido ofreció propuestas elaboradas al respecto de la educación indígena. El PRI proponía duplicar la jornada escolar, ofrecer computadoras y clases de inglés en todas las primarias, el Partido de la Revolución Democrática (PRD) defendía la gratuidad de la educación y el aumento del gasto educativo, y el Partido Acción Nacional (PAN) también prometió elevar el gasto educativo.

El nuevo secretario de la SEP, Reyes Tamez Guerra reconoció que no se puede educar a los 32 millones de mexicanos en rezago, entre analfabetos y sin educación básica. De allí que se desprenda un interés en la educación de los más marginados, pues dentro de los grupos vulnerables de la población se considera a la población indígena en primer lugar. El presidente Fox anunció en San Cristóbal de las Casas la creación de una nueva Coordinación General de Educación Intercultural Bilingüe (Latapí, 2001, pp. 43-44). Así, el gobierno muestra una aparente voluntad y cierto reconocimiento a las demandas en materia educativa de los pueblos indígenas. La propuesta intercultural bilingüe parece hacer suyos los principios del respeto y fomento de las diversas culturas que existen en el país, pero no se incorporó a las comunidades indígenas -con su propia visión educativa- al proyecto de nación multiétnica.

\section{La escritura wixáritari}

Uno de los problemas que enfrentó la edición de libros antes de 1984 fue la ausencia de una escritura que por convención se pudiera generalizar y por lo tanto convertir en una forma de comunicación entre los hablantes del wixárika. No se habían planteado signos para representar sonidos ajenos al español, formas de separar palabras ni reglas de puntuación acordes a la lógica y ritmo de su lengua. De esta manera los educadores del ILV, el clérigo del catecismo y los autores de los 
primeros libros de texto de la SEP, escribían cada uno como imaginaban se podía trasmitir lo que escuchaban. Actualmente existe una propuesta de escritura y gramática que se plantea y enseña desde la Universidad de Guadalajara (UdeG).

Lo reciente de esta historia nos genera el problema de la poca bibliografía al respecto: no hemos encontrado una historia de su aparición y devenir. Por otro lado, su aún corta existencia también nos permite reconstruir los hechos a través de sus participantes. ${ }^{9}$ A continuación, algunos de los hechos.

Un encuentro en 1984 entre Fernando Leal y Rocío Echeverría, fundadora de la Casa Huichol para enfermos y familiares de enfermos wixáritari, podría marcar el inicio de la construcción de una escritura wixárika tendiente a la estandarización. Echeverría llevaría a Leal al encuentro solicitado con los wixáritari a cambio de que realizara un encargo que tenía del Instituto Mexicano del Seguro Social (IMSS): un diccionario wixárika-español para el uso de los médicos con pacientes wixáritari, con quienes no se podían comunicar por ser ambos monolingües en wixárika o en español. Para Leal la elaboración de un diccionario no cumpliría con las necesidades de los médicos, ya que un compendio de palabras no genera comunicación; su propuesta fue hacer una lista de frases relevantes en el contexto médico/paciente. Con ayuda de un médico general y en contacto con las condiciones locales, elaboró 300 preguntas. La idea de Leal era que los médicos que subían a la sierra lo hacían por pocos meses, estaban saturados de trabajo y no llegarían a dominar el idioma; las preguntas del manual de consulta entonces debían ser pronunciables por los médicos y comprendidas por los pacientes. A la vez estas preguntas debían ser respondidas con un sencillo sí o no que el médico pudiera comprender.

En la primera Casa Huichol en las calles de Arista y Tepic, en la ciudad de Guadalajara, Fernando Leal con Mauricio Montellano De la

9 Se entrevistó a Fernando Leal, doctor en Filosofía, Filología y Lingüística por la Universidad de Colonia, Alemania, y a Xitákame Julio Ramírez De la Cruz, maestro en Lingüística Aplicada por la UdeG; ambos son investigadores de la UdeG y participantes activos en el proceso de escriturar la lengua wixárika. 
Cruz, secretario de la comunidad de San Andrés Cohamiata, realizaron un doble trabajo de traducción: Montellano traducía las preguntas a su lengua y Leal las traducía a signos del Alfabeto Fonético Internacional.

El proceso de asignar una escritura a la lengua wixárika comenzó con la identificación de los símbolos en el Manual Internacional utilizado por los lingüistas para todas las lenguas ágrafas. Sin embargo, dada la dificultad de algunos de esos signos para los hispanohablantes, Leal hizo ciertas modificaciones. La lengua wixárika tiene pocos sonidos que el alfabeto hispano no pueda representar, por lo tanto solo se agregaron tres símbolos: la $\dot{+}$ que Leal en un principio construyó en la máquina de escribir con la $i$ y la tecla del guión. Otras letras que se consideraron importantes fueron la $x$ para representar el sonido fuerte de la $r r$ y la $w$ que al mismo tiempo se podía pronunciar como $v$ o como doble $v$ según la pronunciación de cada comunidad. La primera letra fue invento de Leal de acuerdo a las posibilidades tecnológicas en 1984; la segunda y la tercera tienen que ver con una negociación entre los wixáritari de diferentes regiones quienes deseaban asignar en la escritura sus acentos particulares. Fernando Leal, con la participación de Paula Gómez López y Silvia Leal Carretero, ambas investigadoras de la UdeG, realizaron una sencilla publicación titulada Manual para Uso de Médicos con Pacientes de Habla Huichola, distribuida en 1984.

En un segundo momento, Leal pudo constatar que en la Sierra Huichola no eran utilizados los libros de texto gratuito en huichol, publicados y distribuidos por la SEP en 1981. Estos libros que tenían como objeto facilitar la enseñanza de la lecto-escritura a los niños wixáritari eran desechados por los maestros. Leal encontró que los signos de la transcripción no eran el problema sino la separación de las palabras. Los textos eran ilegibles por los hablantes de wixárika, ya que no correspondían a nada que pudiera reconocerse como ideas completas. El problema radicaba en que no había un concepto de "palabra" en la lengua wixárika y los autores de estos libros de texto gratuitos se habían visto en la necesidad de recortar el discurso y hacer pausas a su antojo.

La necesidad de encontrar las fronteras de las "palabras" llevó a Leal al encuentro con hablantes del wixárika en Santa Catarina y San Sebastián, quienes decían hablar diferente en cada comunidad. Así pues, se organizó en la UdeG, en 1987, un encuentro con wixáritari de todas las 
comunidades para discutir el amplio problema de las variantes. El objetivo era crear una lengua escrita que satisfaciera a todos. Tras una semana de largas disertaciones no se lograron conciliar las distintas propuestas; quedaba al descubierto que la lengua también es un acto político y que ningún grupo iba a permitir ser dominado por la lengua de otro.

La propuesta de una escritura nueva no tiene aceptación inmediata ni se puede suponer que los hablantes del wixárika y alfabetizados en español, puedan automáticamente leer el wixárika escrito y recién fundado. En 1991 el lector wixárika se veía:

Confrontado con tres tipos de escritura: la de la SEP (en el libro de primer grado), la elaborada según criterios más estrictamente lingüísticos por la UdeG y la que los propios wixáritari consideran más apta para su lengua. ... El desacuerdo entre las tres variedades ortográficas existe principalmente en cuanto acentos, los apóstrofes y también la separación de palabras (Steckbauer, 1993, p. 449).

\section{La estandarización de la escritura}

En el caso de la escritura wixárika como otros casos de escrituras impuestas (el español como segunda lengua, el huave, etc.) los hablantes se enfrentan con grandes dificultades para leer y escribir. Cuando la escritura surge desde los hablantes, las convenciones se construyen con mayor facilidad. El wixárika no ha encontrado ese acuerdo ya que la iniciativa de los profesores hablantes y la propuesta académica aún se debaten. Probablemente los wixáritari construirán sus convenciones a partir de usos concretos y necesidades imperiosas. Este es el caso de los jóvenes indígenas huaves en Facebook, quienes construyen una escritura común y por lo tanto construyen un proceso nuevo de estandarización. ${ }^{10}$

En este momento ciertos problemas de fondo han sido superados:

- Las letras $x, \dot{+}, w$, se han aceptado en casi todos los casos, si bien no hay aún homogeneidad en el uso de las mayúsculas de estas letras.

10 Samuel Herrera, desde el Instituto Nacional de Lenguas Indígnas (INALI) realiza actualmente una investigación sobre la convención y el uso juvenil huave en Facebook. 
- La acentuación gráfica puede o no aparecer y no altera el significado para los lectores wixáritari que la conocen.

- Queda por encontrar una división de palabras que por convención sea aceptada.

- La escritura académica desde las reglas subyacentes o las propuestas populares según el oído son aún debatidas.

- Hay libros que usan una escritura; hay otros que se inclinan por otra, y los hay que combinan ambas.

LOS LIBROS PARA WIXÁRITARI

TABLA 1

LAS EDITORIALES Y NÚMERO DE TÍTULOS PUBLICADOS PARA LOS WIXÁRITARI

Editoriales Títulos

Secretaría de Educación Pública (SEP) 22

Universidad de Guadalajara (UdeG)

14

Instituto Lingüístico de Verano (ILV)

Instituto Tecnológico y de Estudios Superiores de Occidente (ITESO) 7

Consejo Nacional para la Cultura y las Artes (CONACULTA) 4

Fundación Ford

Otros: Gobiernos de los estados de Jalisco, Nayarit y

el Distrito Federal

S/D

Fuente: Elaboración propia.

La marca editorial nos permite observar que la edición wixárika es realizada por instituciones oficiales estatales, de la Iglesia y editoras sin fines de lucro como la Fundación Ford, el Centro de Derechos Humanos Agustín Pro, etc. La SEP encabeza la lista con 22 títulos, le sigue la UdeG con 14 y el ILV con 8. Se mencionan los casos en los que aparece la institución como editora única o como coeditora, por lo que el número total de menciones supera los 55 títulos del corpus. Los libros dirigidos a los wixáritari son en todos los casos gratuitos. En otras palabras el costo del libro que lee el wixáritari no es sufragado por él mismo. 
En México la gratuidad del libro tiene una larga historia que data por lo menos de la fundación de la SEP en 1921. La propuesta alfabetizadora primero y promotora de la lectura después, ha consistido en distribuir masivamente libros gratuitos para todos los mexicanos. Esta estrategia ha sido especialmente favorable para las familias de muy escasos ingresos que si no fuera de esta manera, el libro les sería un objeto desconocido.

Los libros para indígenas pertenecen a esta política pública. En los casos de editoriales comerciales como Diana y Petra, publican una primera edición corta para venta en los circuitos comerciales y una segunda de tiraje masivo en coedición con la SEP que se distribuye gratuitamente en escuelas públicas. Las dos únicas ediciones autónomas a la tendencia de coedición son la del Centro de Derechos Humanos Agustín Pro y la del Centro Indígena Huichol A.C., ambas publicaciones digitales.

Del corpus de 55 títulos, 26 fueron editados en el Distrito Federal; 23 en Jalisco, 1 en Zacatecas, 1 en Nayarit y 1 en Chiapas. La influencia del centralismo de "la ciudad letrada" se deja ver en este proceso editorial. Pero también se puede ver el interés regional donde sobresalen los 19 títulos hechos en Jalisco en los que aparece la UdeG o el Instituto Tecnológico y de Estudios Superiores de Occidente (ITESO) como editor o coeditor.

TABLA 2

NÚMERO DE TÍTULOS Y EDITORIALES, POR DÉCADAS, QUE PUBLICAN PARA LOS WIXÁRITARI

Década Títulos, ediciones

Editoriales $\mathrm{y}$ reediciones

\begin{tabular}{|c|c|c|}
\hline 1906 & 1 & Asilo del Sagrado Corazón de Jesús \\
\hline 1947 & 1 & $\begin{array}{l}\text { Instituto Lingüístico de Verano/Secretaría de } \\
\text { Educación Pública }\end{array}$ \\
\hline $1950-1959$ & 5 & $\begin{array}{l}\text { Instituto Lingüístico de Verano/Secretaría de } \\
\text { Educación Pública }\end{array}$ \\
\hline $1960-1969$ & 3 & Instituto Lingüístico de Verano \\
\hline $1970-1979$ & 1 & Instituto Lingüístico de Verano \\
\hline 1980-1989 & 8 & $\begin{array}{l}\text { Instituto Lingüístico de Verano/Secretaría de } \\
\text { Educación Pública, Secretaría de Educación }\end{array}$ \\
\hline
\end{tabular}




\begin{tabular}{|c|c|c|}
\hline Década & $\begin{array}{c}\text { Títulos, ediciones } \\
\text { y reediciones }\end{array}$ & Editoriales \\
\hline & & $\begin{array}{l}\text { Pública, Casa de Salud Huichol/UdeG, UdeG/ } \\
\text { Universidad Autónoma de Nayarit (UAN) }\end{array}$ \\
\hline 1990-1999 & 11 & $\begin{array}{l}\text { Secretaría de Educación Pública, Universidad } \\
\text { de Guadalajara, CONACULTA, Diana }\end{array}$ \\
\hline 2000-2009 & 28 & $\begin{array}{l}\text { Secretaría de Educación Pública, Universidad } \\
\text { de Guadalajara, Asociación de escritores en } \\
\text { lengua indígena/Gobierno del Distrito Fede- } \\
\text { ral, Universidad Pedagógica Nacional (UPN)/ } \\
\text { SEP, Gobierno del Estado de Jalisco, Fundación } \\
\text { Ford, CONACULTA, CONACYT, Petra, ITESO }\end{array}$ \\
\hline $2010-2011$ & 3 & $\begin{array}{l}\text { CONACULTA, Universidad Autónoma de Chia- } \\
\text { pas (UNACH)/Centro de Estudios Jurídicos y } \\
\text { Sociales (CENEJUS) }\end{array}$ \\
\hline
\end{tabular}

Fuente: Elaboración propia.

En la Tabla 2 podemos ver que salvo el catecismo de 1906, el resto de las publicaciones hechas para wixáritari hasta 1979 se realizaron exclusivamente por el ILV o con SEP en coedición; aún aparece una pre-cartilla y el Nuevo Testamento del ILV en la década que sigue. En estos datos también observamos que la década de 1980 marca un parteaguas en la edición para wixáritari por varios motivos. En estos años se propone construir una escritura del wixárika donde por convención se pueda generalizar su uso. En 1984 se publica el primer libro con esta propuesta y es también con este libro que la UdeG inaugura su edición para wixáritari.

En la década de 1990 se observa la salida definitiva del ILV del campo de la edición educativa para los wixáritari. La SEP se especializa con 6 nuevos títulos en libros de texto bilingües y la UdeG amplía su edición a 5 títulos de rescate de la cultura. La tendencia se acentúa en la década de 2000 y se agrega la edición de nuevas expresiones wixáritari narrativas y fotográficas. Aparecen en el Departamento de Estudios de la Comunicación Social (DECS) de la UdeG, 3 títulos dialógicos realizados entre wixáritari y no wixáritari con una propuesta intercultural distinta. Estos libros muestran explícitamente la participación indígena 
y no indígena en una búsqueda de equidad discursiva. En el primer año de la presente década se detecta la reedición de un libro de literatura wixárika de 1994 y 2 nuevos textos dialógicos. Los libros de texto para el bachillerato, publicados por el ITESO, fueron realizados con los maestros wixáritari del primer bachillerato intercultural fundado por los habitantes de la Sierra Huichola.

TIRAJES

TABLA 3

CLASIFICACIÓN DE TÍTULOS POR TIRAJES

\begin{tabular}{lc}
\hline \multicolumn{1}{c}{ Tirajes (número de ejemplares) } & Títulos \\
\hline Especializado 1-500 & 14 \\
Comercial 501-3,000 & 15 \\
Éxito editorial 3,001-10,000 & 9 \\
Masivos 10,001-100,000 & 4 \\
S/D & 13 \\
\hline
\end{tabular}

Fuente: Elaboración propia.

Los tirajes de los libros para los wixáritari oscilan entre los $100 \mathrm{y}$ los 97 mil 803 ejemplares. Se han nombrado y clasificado los títulos en cuatro tipos de tirajes que corresponden a distintas políticas editoriales en México. Este dato nos acerca a los propósitos de las editoriales con relación al alcance de los títulos: El tiraje especializado alcanza a un número limitado de lectores y nos habla de libros concebidos para un lector particular, definido por gustos y necesidades de libros exclusivos. El ILV, la SEP, la Casa de Salud Huichol y universidades como la UdeG, la UNACH, el ITESO y el CONACYT han editado con esta política.

El tiraje comercial en México pretende llegar a un público que lee libros de temas expertos, aunque los libros de tiraje comercial tienen más que ver con fines de comercialización. Con relación a los libros para wixáritari, si bien sabemos que no se venden, la tendencia es similar. La mayor parte de los títulos que analizamos se encuentran en este rango de tiraje y los editan editoriales como la UdeG, CONACULTA, Fundación Ford y editoriales comerciales como Diana y Petra. 
Entre los tirajes especializados y comerciales, es decir, menos de 3 mil ejemplares, se encuentran 29 de los 55 títulos analizados. Pareciera que el criterio de más de la mitad de los títulos, es cubrir un servicio de lector reducido.

Los éxitos editoriales son libros que abordan intereses más amplios; con este tipo de tiraje nos referimos a los títulos editados en más de 3 mil 001 y menos de 10 mil 001 ejemplares. Estos tirajes llevan la intención de llegar extensamente a un público amplio.

Finalmente los tirajes masivos llegan a públicos extensos -ya sea por ser éxitos comerciales inéditos o por pertenecer a políticas públicas de interés nacional- y responden a una política cultural general con intenciones de abarcar un público nacional extenso. En este rubro solo la SEP tiene capacidad de editar masivamente para wixáritari. En los 4 títulos que responden a este tiraje aparecen como coeditores la UdeG, y las editoriales Petra y Diana. En 3 de los 4 casos de tirajes masivos, los libros se distribuyen en el circuito de la SEP a niños wixáritari y no wixáritari.

\section{GÉNEROS}

\section{TABLA 4}

NÚMERO DE TÍTULOS POR GÉNERO

\begin{tabular}{lc}
\hline Género & Títulos \\
\hline Cultura wixárika & 17 \\
Libros de texto (escolares) & 23 \\
Informativos (adultos) & 4 \\
Religioso (catecismo y Nuevo Testamento) & 2 \\
Diccionario & 4 \\
Cartilla & 5 \\
\hline
\end{tabular}

Fuente: Elaboración propia.

En una clasificación formal casi todos los libros para wixáritari corresponden al género didáctico, un género "especial, que queda fuera de la verdadera literatura porque está ordenado a un fin y no es por consiguiente, literatura autónoma" (Kayser, 1976, p. 440). Sin preten- 
der valorar la forma expresiva de estos libros, podemos sin embargo analizar sus objetivos, en los que se observan diferentes modalidades: el autor se dirige al destinatario para educarlo, informarle, adoctrinarlo, catequizarlo o cultivarlo. Son educadores, eclesiásticos e investigadores que se dirigen a un público principalmente escolar. Los libros de texto y algunos de los títulos de cultura wixárika pertenecen a este grupo. Los libros informativos, religiosos y las cartillas, dirigidas a un público adulto también conllevan un fin pedagógico frente a la salud, la alfabetización y la espiritualidad.

Los libros de texto, los informativo/educativos, así como los religiosos son naturalmente imperativos y difieren de los demás géneros en que no pueden ser sometidos a un diálogo. El expresar "haz esto", "aprende eso", "actúa así" no tienen las mismas propiedades que las declaraciones: "hacer esto", "aprender eso", "actuar así" o menos aún pueden ser convertidas en interrogaciones como "¿hacer esto?”, “¿aprender eso?”, “¿actuar así?”. En nuestro corpus se observa una firme intención de educar al indígena en 45 títulos del total de los 55 títulos.

Hay también ciertos textos que escapan el género educativo puro, son aquellos que buscan entablar comunicación. Los diccionarios si bien pueden ser limitados compendios de palabras y sus significados, tienen la doble intención de reglar el significado de las palabras y a la vez facilitar la traducción. Aquí también pertenece el Manual para Uso de Médicos que a partir de una lista de preguntas, a pesar de lo limitado de las respuestas, se inclina a una mejor comprensión entre los interlocutores.

Caso distinto son los libros del corpus que se denominan entreculturales; estos exponen una alternativa a la comunicación pedagógica unidireccional a partir del diálogo entre las culturas. El objetivo de los 5 libros "bidireccionales" es mostrar en conflicto o negociadas -según sea el caso- las formas distintas de abordar la cultura desde la voz propia con relación horizontal frente a la otra cultura. Se muestran distintas maneras de verse a sí mismo y al otro, de concebir la oralidad y la escritura, así como posiciones sobre cómo aparecer en la fotografía, o sobre los temas de la belleza, la salud, el trabajo, la justicia y la historia nacional.11

11 Véanse Corona (2003, 2007, 2011); Pérez-Daniel y Corona (2011); Salvador y Corona (2002). 


\section{LENGUA}

\begin{tabular}{lc}
\multicolumn{2}{c}{ TABLA 5 } \\
LENGUA DE LOS LIBROS \\
\cline { 2 - 3 } Lenguas & Títulos \\
\hline Bilingüe & 30 \\
Wixárika & 15 \\
Español & 7 \\
s/d & 3 \\
\hline
\end{tabular}

Fuente: Elaboración propia.

Los libros son en su mayoría bilingües en español y wixárika. Algunos de los títulos fueron concebidos en español primero y posteriormente traducidos para interpelar al wixárika en su propia lengua. Los libros bilingües pueden ser leídos también por los lectores no bilingües a quién explícitamente también se dirigen. El caso de los libros del ITESO - con una propuesta pedagógica novedosa hecha por maestros wixáritari y asesores mestizos en temas científicos- fueron escritos en español por ser la lengua en que ambos grupos escribían mejor. Mencionan que les faltaron recursos económicos para traducir la amplia obra.

Si bien con propósitos distintos, el catecismo primero y los diccionarios y el manual médico después, muestran explícitamente la función puente entre los hispanohablantes no hablantes de lengua wixárika y los wixáritari monolingües en su propia lengua.

Algunos libros educativos para la formación intercultural de ambos -wixáritari y no wixáritari- hacen patente el bilingüismo como forma de comunicación con los otros. Estos libros no presuponen que los interlocutores indígenas no lean el español o que los no indígenas aprendan el wixárika, ya que se dirigen al lector dentro del sistema escolar, sino que se pretende dar visibilidad a la lengua indígena. Algunos de estos textos podrían definirse en lo políticamente correcto sin embargo otros buscan más genuinamente la horizontalidad de las lenguas.

Los libros monolingües en wixárika son especialmente valorados por los profesores bilingües wixáritari, lo cual se muestra en los tres títulos reeditados desde 1992 hasta 2001 y que aún se usan en las primarias 
wixáritari. Otros títulos son escritos por los wixáritari con la intención de construir material de lectura en su propia lengua y con el fin de que tenga sentido aprender a escribirla. En este caso están los libros de los wixáritari que pertenecen al género cultural donde se rescatan canciones, historias y rituales antiguos contados por los ancianos de las comunidades. La complejidad de la autoría en los casos de los libros interculturales ha sido tratada a fondo por Rebeca Pérez-Daniel (2012). La autora encuentra que algunos de los textos educativos interculturales dirigidos a niños wixáritari son escritos por autores que no conocen la cultura y que terminan por reproducir identidades estereotipadas. En contraste, el proceso de autoría "entre voces" requiere de planteamientos teóricos que la autora explica:

Las autorías interculturales suponen que la visibilización basta para las buenas relaciones. ... Y lo que es más complicado aún, suponen que la exposición de un sujeto es la visibilización de un grupo. ... En el proyecto Entre Voces, los docentes tenían un interés concreto: el intercambio para el mutuo reconocimiento. Por tanto, era necesario que la exposición fuera hecha de lugares comunes, pero de lugares comunes para cada quien. Así, el contraste entre uno y otro evidenciaba el contraste entre un contexto y otro. ... Por tanto, lo que da la utilidad de la visibilización no es la visibilización en sí, sino el sistema de significación que se utiliza como referente (pp. 61-62).

De esta manera, el corpus de textos en este artículo pertenecen en su mayoría a autores no wixáritari. De este grupo se excluyen los libros escritos en wixárika por wixáritari, así como los libros entreculturales que explícitamente indican el tipo de diálogo entre los autores de cada cultura y están escritos en ambas lenguas.

\section{¿INTEGRACIÓN, EXCLUSIÓN O EMANCIPACIÓN?}

¿Qué hay en Jalisco para los wixáritari a casi 200 años de la primera Constitución, que tenía como objetivo propagar la escritura para "hacer ciudadanos"?, ¿son los libros editados para ellos, medios de integración, de exclusión o de emancipación?

La producción de la SEP como principal editora para indígenas comporta aciertos importantes. Los tirajes pequeños que contrastan con los 
tirajes de las políticas educativas generales permiten, a quienes hablan lenguas distintas al español, acceder al libro. Si bien se conocen mejor las ediciones millonarias, es de resaltar la importancia de las especializadas. Otro logro fundamental para la educación indígena ha sido la publicación de libros exclusivamente laicos. Relacionar la educación y el libro con la religión, tendría efectos desastrosos para las culturas indígenas.

Por otro lado, los libros muestran que en 90 años que tiene de existir la SEP, no se han realizado propuestas educativas radicalmente distintas. Relaciones de poder desiguales están presentes en el objetivo de alfabetizar, castellanizar y educar. No hay voluntad real por conocer las culturas indígenas. Las lenguas se rescatan como rasgo distintivo pero los testimonios, prácticas de salud y belleza, saberes económicos, políticos, etc. de los múltiples pueblos indígenas contemporáneos, son ignorados. Sus historias se reproducen desvinculadas de su contexto étnico y cosmogónico por lo que resultan sin consistencia ni continuidad, simples e infantiles.

Los momentos históricos marcan sutiles diferencias, pero la reciente interculturalidad como proyecto educativo no conlleva cambios sustantivos; no deja de ser una manera del Estado-nación de moverse con la globalización. Aunque surjan en los libros las nuevas identidades indígenas, no son ajenos a la intención de fabricar sujetos nacionales. Mientras el libro educativo no tenga su origen en la voz de los pueblos indígenas o esta sea descalificada y mantenida al margen, la experiencia educativa nacional no será completa ni será exitosa.

La SEP define en muchos aspectos lo que el país lee. Muchos indígenas leerán en su vida únicamente lo que la SEP les ofreció en su infancia, por lo tanto conocerán el libro desde la definición de temas, autores, géneros, tamaños, contemplados por las políticas editoriales de cada periodo de gobierno. Por ello faltan otros equilibrios, como la presencia equitativa de las miradas indígenas en los libros para indígenas y para no indígenas.

También es de resaltar la participación de la UdeG y posteriormente del ITESO, ambas universidades de Jalisco, que desde lo local han diversificado sus metas: de la edición de libros exclusivamente de investigación y docencia para públicos académicos, a la edición para lectores wixáritari. La UdeG se incorpora a la edición de los libros para 
wixáritari desde el Departamento de Estudios en Lenguas Indígenas y posteriormente desde el DECS. El ITESO publica con los wixáritari desde su Departamento de Educación. Sin embargo extrañamos una ausencia local fundamental: la de la SEP del estado de Jalisco. Siendo dicho estado sede de varios pueblos indígenas y asiento ancestral de los wixáritari, el abandono en el tema de las publicaciones para indígenas, es preocupante. Instituciones como INI-CDI Jalisco, tampoco muestran un proyecto editorial para indígenas del estado.

La producción de libros en México hoy nos exige pensar su relación con el mundo "en medio de una doble tormenta", como nombra Jesús Martín-Barbero la situación latinoamericana. Esto es, encontrar formas de vida en "la encrucijada" entre las lógicas de la globalización y la centralización, y las múltiples historias propias de los muchos diversos mexicanos.

En este sentido, lo nacional como nunca antes, debe buscar formas de hacerse cargo de las culturas nacionales y locales, y de la oferta global. Es inminente una nueva historia nacional frente a la debilidad que han mostrado las instituciones (como la familia, los medios masivos de comunicación y el mercado) para regular y potenciar estas fuerzas que hoy nos envuelven como tormentas.

En más de un siglo se han producido libros educativos para wixáritari con intención de integrarlos al proyecto del Estado nacional. Sin embargo, se les ha excluido de la decisión del proyecto mismo de nación. Producir libros indígenas en diálogo con la sociedad mayoritaria, que tampoco es monolítica y homogénea, puede ser una vía emancipatoria. En este sentido recojo la importancia de la producción de muchos libros para wixáritari de autores wixáritari y autores no wixáritari que relaten fragmentos diversos de este conjunto que llamamos México.

\section{Bibliografía}

Bravo Ahuja, V. (1976). La obra educativa. México: SEP.

Castañeda, C. (1984). La educación en Guadalajara durante la Colonia 1552-1821. Guadalajara, Jalisco, México: El Colegio de Jalisco/ El Colegio de México.

Constitución Política del Estado Libre de Xalisco. (1824). México. 
Corona Berkin, S. (2003). Álbum de fotografía huichol. Libros del Rincón. México, D.F.: SEP.

Corona Berkin, S. (2007). Entre voces. Fragmentos de educación entrecultural. Guadalajara, Jalisco, México: UdeG.

Corona Berkin, S. (2011). Postales de la diferencia. La ciudad vista por fotógrafos wixáritari. Culturas Populares de México. México, D.F.: CONACULTA.

Corona Berkin, S. \& De Santiago, A. (2011). Niños y libros. Publicaciones infantiles de la SEP. México: SEP.

Florescano, E. (1999). Memoria indígena. México: Taurus.

Gómez, P. \& Ramírez De la Cruz, X. (1989). Antología narrativa huichola. Guadalajara, Jalisco, México: UdeG.

Heath, S. B. (1986). La política del lenguaje en México de la Colonia a la Nación. México: INI.

Kayser, W. (1976). Interpretación y análisis de la obra literaria. Madrid, España: Gredos.

Labra, A. (Comp). (1985). Narciso Bassols. México: Crea/Terra Nova.

Latapí Sarre, P. (2001, 28 de enero). El primer reto: la educación indígena en México. Revista Proceso, 1265, 43-44.

Leal, F., Gómez, P. \& Leal, S. (1984). Manual para uso de médicos con pacientes de habla huichola. Cuadernos de la Maestría en Salud Pública 6. Guadalajara, Jalisco, México: UdeG.

Lévi-Strauss, C. (1992). Tristes trópicos. Madrid, España: Paidós.

Limón Rojas, M. (2000). Memoria del quehacer educativo 1995-2000 (Tomo I). México: SEP.

Martín-Barbero, J. (1987). De los medios a las mediaciones. Comunicación, cultura y hegemonía. Barcelona, España: Gustavo Gili.

Martín-Barbero, J. (2001). Imaginarios de nación. Pensar en medio de la tormenta. Bogotá, Colombia: Ministerio de Cultura.

Morales Meneses, E. (1986). Tendencias educativas 1911-1934. México: Centro de Estudios Educativos/UIA.

Pérez-Daniel, R. (2010, enero/junio). El estudio de los libros de texto de educación intercultural como vía de aproximación para la construcción del espacio público. Por un esclarecimiento de los procesos de autoría. REDHES, Revista de Derechos Humanos y Estudios Sociales, 3 (II), 85-110. 
Pérez-Daniel, R. \& Corona, S. (2011). Wiixaaritaari Kiekaritsie Mepikiinetiwe (Los wixáritari viajan a la ciudad). México, D.F.: CENEJUS/UNACH/CONACYT/UCICH.

Pérez-Daniel, R. (2012, diciembre/mayo). Los procesos de autoría en la creación de libros educativos interculturales: debate y reflexión desde un ejercicio de réplica de la metodología de Entre-Voces. Pueblos y Fronteras, 12 (7), 39-66.

Plan Nacional de Desarrollo 1989-1994 (1989, 31 de mayo). México.

Rama, A. (1984). La ciudad letrada. Hanover, EE.UU.: Editorial Del Norte.

Ramírez De la Cruz, X. (1995). Nosotros los huicholes. En J. L. Iturrioz (Coord.), Reflexiones sobre la identidad étnica. Guadalajara, Jalisco, México: UdeG.

Ramírez De la Cruz, X. (2006, enero/marzo). Política lingüística y educación bilingüe bicultural: problemas y necesidades de la región huichol en México. América Indígena, 1 (LXII).

Revista Educación (1923, 1 de mayo). 1 (2).

Salvador, A. \& Corona, S. (2002). Xalapa taniuku maye'uxa meripai hik+timieme (Nuestro libro de la memoria y la escritura). Guadalajara, Jalisco, México: UdeG.

Steckbauer, S. (1993, 7-11 de septiembre). Actes de la "decouverte" de langues et des écritures d'Amerique. París, Francia: Colloque International.

Torres Bodet, J. (1946). La obra educativa en el sexenio 1940-1946. México: SEP.

Vasconcelos, J. (1952). El desastre. México: Ediciones Botas.

Zedillo Ponce de León, E. (1997, 1 de septiembre). Avances y retos de la nación. México.

Fecha de recepción: 10/05/12. Aceptación: 01/06/12. 